\title{
Single scattering by realistic, inhomogeneous mineral dust particles with stereogrammetric shapes
}

\author{
H. Lindqvist ${ }^{1}$, O. Jokinen ${ }^{2}$, K. Kandler ${ }^{3}$, D. Scheuvens ${ }^{3}$, and T. Nousiainen ${ }^{1,4}$ \\ ${ }^{1}$ University of Helsinki, Department of Physics, Helsinki, Finland \\ ${ }^{2}$ Aalto University, Department of Real Estate, Planning and Geoinformatics, Espoo, Finland \\ ${ }^{3}$ Technische Universität Darmstadt, Inst. für Angewandte Geowissenschaften, Darmstadt, Germany \\ ${ }^{4}$ Finnish Meteorological Institute, Helsinki, Finland
}

Correspondence to: H. Lindqvist (hannakaisa.lindqvist@ helsinki.fi)

Received: 23 May 2013 - Published in Atmos. Chem. Phys. Discuss.: 9 July 2013

Revised: 7 November 2013 - Accepted: 12 November 2013 - Published: 6 January 2014

\begin{abstract}
Light scattering by single, inhomogeneous mineral dust particles was simulated based on shapes and compositions derived directly from measurements of real dust particles instead of using a mathematical shape model. We demonstrate the use of the stereogrammetric shape retrieval method in the context of single-scattering modelling of mineral dust for four different dust types - all of them inhomogeneous - ranging from compact, equidimensional shapes to very elongated and aggregate shapes. The three-dimensional particle shapes were derived from stereo pairs of scanningelectron microscope images, and inhomogeneous composition was determined by mineralogical interpretation of localized elemental information based on energy-dispersive spectroscopy. Scattering computations were performed for particles of equal-volume diameters, from $0.08 \mu \mathrm{m}$ up to $2.8 \mu \mathrm{m}$ at $550 \mathrm{~nm}$ wavelength, using the discrete-dipole approximation. Particle-to-particle variation in scattering by mineral dust was found to be quite considerable and was not well reproduced by simplified shapes of homogeneous spheres, spheroids, or Gaussian random spheres. Effective-medium approximation results revealed that particle inhomogeneity should be accounted for even for small amounts of absorbing media (here up to $2 \%$ of the volume), especially when considering scattering by inhomogeneous particles at size parameters $3<x<8$. When integrated over a log-normal size distribution, the linear depolarization ratio and singlescattering albedo were also found to be sensitive to inhomogeneity. The methodology applied is work-intensive and the light-scattering method used quite limited in terms of size parameter coverage. It would therefore be desirable to find a
\end{abstract}

sufficiently accurate but simpler approach with fewer limitations for single-scattering modelling of dust. For validation of such a method, the approach presented here could be used for producing reference data when applied to a suitable set of target particles.

\section{Introduction}

Atmospheric mineral dust scatters and absorbs solar radiation and emits thermal radiation, contributing to the radiation balance of the Earth-atmosphere system and influencing atmospheric remote-sensing observations. Dust aerosol particles play an important role as freezing nuclei in the global water cycle and as nutrients in various ecosystems, but they also pose potential respiratory and aviation hazards (Goudie and Middleton, 2006). Local and global concentrations of dust are monitored through remote-sensing methods, such as lidars or satellite retrievals, based on the radiation scattered by ensembles of single dust particles. The scattering properties of different, individual dust particles are highly dependent on their physical properties, and this relation can be established either by measuring the single-scattering properties for particles whose physical properties are known, or computing them with a single-scattering model. For single particles of a few micron size, which are most abundant in mineral dust around the globe (Reid et al., 2008; Formenti et al., 2011), such measurements are not yet available, even though scattering measurements exist for several dust ensembles collected from various locations (Muñoz et al., 2012). 
This leaves single-scattering modelling as the only presently available alternative.

There are two distinct parts in single-scattering modelling: (i) modelling the shape and composition of the particle and (ii) modelling scattering, i.e. the interaction between material and electromagnetic radiation. These parts are not wholly independent, because most light-scattering methods have inherent limitations in what kind of model particles and the level of details they can be applied to, and because the singlescattering properties depend on the characteristics of the model particles. This dependence can be quite complex, so it is not necessarily obvious what kind of assumptions or simplifications can be made in the description of the model particles without significantly altering the single-scattering properties (e.g. Nousiainen et al., 2012). Mineral dust particles may differ in mineralogical composition, shape, smallscale surface structures and internal inhomogeneity, posing challenges on the development of representative models. Ideally, model particles should be representative of the real dust particles in all characteristics relevant for scattering. If such model particles are used together with a scattering model that provides a rigorous and numerically accurate solution to the Maxwell equations, the obtained single-scattering properties are obviously representative of the real dust particles.

The dust models proposed thus far, even the more realistic ones, are mathematical shape models that have been validated through comparing computed scattering by an ensemble of these shapes to laboratory-measured scattering properties of an ensemble of real mineral dust particles (e.g. Bi et al., 2009, 2010; Dubovik et al., 2006; Ishimoto et al., 2010; Merikallio et al., 2011; Nousiainen et al., 2011b). Despite the morphological simplicity of some of the models, these approaches have produced a good agreement between simulated and measured light-scattering properties. However, because different size-shape distributions of particles, especially those consisting of such complex particles as mineral dust, have been found to scatter light surprisingly similarly (Nousiainen et al., 2012), it is not necessary that the physical properties of the model particles correlate in any clear way with the corresponding properties of the target particles in spite of similar scattering (Nousiainen et al., 2011a). Still, there is nothing to guarantee that the optical equivalence holds for parameter combinations not tested, e.g. for different size distributions or at different wavelengths. For example, Merikallio et al. (2011) showed that to optimally reproduce scattering by a given dust sample at different wavelengths using spheroidal model particles, different sets of spheroids are needed at different wavelengths. If, on the other hand, a realistic shape model is used, the approach should be free from such concerns.

Here, we choose a new, different approach for singlescattering modelling of dust: we derive the shapes and compositions of four distinct, micron-sized Saharan dust particles directly from observations, and use these shapes to compute the respective single-scattering properties as a func- tion of several particle sizes at visible wavelength. Using the measured physical properties as a starting point frees us from the limitations and characteristics of any predefined shape model, allowing us to also realistically estimate the natural variability in scattering between different dust particles arising from their individual shapes and compositions. The particle shapes are retrieved from scanning-electron microscope (SEM) images by means of stereogrammetry, and their inhomogeneous mineralogical compositions are derived from detailed elemental mappings based on energydispersive spectroscopy. Using these methods, particle inhomogeneity and small-scale surface features can be considered, at least to some extent. Both of these are essential since the single-scattering properties of dust have been estimated to be very sensitive to inhomogeneity, particularly the fraction of hematite (Hu and Sokhi, 2009; Mishra et al., 2012), and to the scale and type of surface roughness (Nousiainen, 2009, and references therein). More generally, surface roughness has been considered as a significant feature of small atmospheric particles with respect to its impact on scattering (see, e.g. Macke et al., 1996; Nousiainen et al., 2003; Sun et al., 2004). The single-scattering results for the inhomogeneous stereogrammetric particles are presented both for monodisperse and polydisperse cases, the latter as integrated over a log-normal size distribution. The consistency of corresponding simplified, homogeneous models (homogeneous stereogrammetric shape, Gaussian random sphere, spheroid, sphere) is also evaluated by comparisons with their scattering properties, both for individual particles and as ensembles.

Finally, we note that the stereogrammetric approach to model scattering by single dust particles more realistically has some analogies to the confocal microscopy measurements method used by Brock et al. (2006) and Orlova et al. (2008) to model light scattering by biological particles: both methods result in a retrieved, three-dimensional model shape for a single particle. Orlova et al. (2008) even validate their computed scattering results by comparisons to measurements performed with a scanning flow cytometer. Indeed, several approaches in addition to flow cytometers (Jacobs et al., 2009; Strokotov et al., 2011) have been taken towards measuring scattering by individual particles: for example, air flow systems measuring two-dimensional scattering patterns (Kaye et al., 2007) and digital holographic imaging (Berg and Videen, 2011).

This paper is structured as follows: relevant light scattering theory is presented in Sect. 2, and the dust particles considered are introduced and depicted in Sect. 3. Section 4 describes the modelling approach from stereogrammetry to three-dimensional shapes and inhomogeneity, and introduces also the simplified models. Scattering results and comparisons are presented and discussed in Sect. 5, while Sect. 6 summarizes and concludes the study. 


\section{Relevant single-scattering theory}

In classical electromagnetic scattering considerations, the properties of radiation can be characterized by four measurable quantities that form the Stokes vector $[I, Q, U, V]^{\mathrm{T}}$. Here, $I$ denotes the total intensity, and $Q, U$, and $V$ describe the polarization state of the radiation. The Stokes vectors for incident and scattered radiation are related through the scattering matrix $\mathbf{S}$, so the scattering matrix specifies how the properties of the radiation are altered in the scattering process. The scattering matrix depends on the wavelength of light $\lambda$, illumination geometry, and the physical properties of the scatterer: shape, size, and composition. Size and wavelength dependencies are linked according to the scale invariance rule (Mishchenko et al., 2002), and therefore $\mathbf{S}$ is often studied as a function of the size parameter $x=2 \pi a_{\mathrm{eq}} / \lambda$, where $a_{\mathrm{eq}}$ is the radius of an equal-volume sphere. When averaging over a large number of orientations, the dependence of $\mathbf{S}$ on the illumination geometry is reduced to one angle only, the scattering angle $\theta$, which specifies the angle between the propagation directions of incident and scattered light. For a single particle, orientation averaging also reduces the number of independent scattering-matrix elements to ten (van de Hulst, 1981):

$$
\left(\begin{array}{c}
I_{\text {sca }} \\
Q_{\text {sca }} \\
U_{\text {sca }} \\
V_{\text {sca }}
\end{array}\right)=\frac{1}{k^{2} r^{2}}\left(\begin{array}{cccc}
S_{11} & S_{12} & S_{13} & S_{14} \\
S_{12} & S_{22} & S_{23} & S_{24} \\
-S_{13} & -S_{23} & S_{33} & S_{34} \\
S_{14} & S_{24} & -S_{34} & S_{44}
\end{array}\right)\left(\begin{array}{c}
I_{\text {inc }} \\
Q_{\text {inc }} \\
U_{\text {inc }} \\
V_{\text {inc }}
\end{array}\right),
$$

where $r$ is the distance from the scatterer and $k$ is the wave number. When averaged over a large number of particles and their mirror particles, six independent scattering-matrix elements remain: $S_{11}, S_{12}, S_{22}, S_{33}, S_{34}$, and $S_{44}$. For unpolarized incident light ( $\left.Q_{\text {inc }}=U_{\text {inc }}=V_{\text {inc }}=0\right)$, the angular distribution of intensity is described by the $S_{11}$ element and can also be characterized by an integral quantity, the asymmetry parameter $g$ given by

$g=\frac{2 \pi}{k^{2} C_{\text {sca }}} \int_{0}^{\pi} \sin \theta \cos \theta S_{11}(\theta) \mathrm{d} \theta$.

Here, $C_{\text {sca }}$ is the scattering cross section that is a measure for the total scattered power.The asymmetry parameter varies from -1 to 1 , depending on the amount of radiation scattered into the backward $\left(\theta>90^{\circ}\right)$ and forward $\left(\theta<90^{\circ}\right)$ hemispheres, respectively. The amount of incident power absorbed by the particle is specified by the absorption crosssection $C_{\mathrm{abs}}$. The total power removed by the particle is described by the extinction cross-section $C_{\text {ext }}$ that is the sum of $C_{\text {sca }}$ and $C_{\text {abs }}$. The relative contributions of scattering and absorption are usually characterized by the single-scattering albedo $\varpi$ given by

$\varpi=\frac{C_{\text {sca }}}{C_{\text {ext }}}$.

When the material is nonabsorbing, $\varpi=1$.
In dust aerosol retrievals, especially in solving the underdetermined lidar equation, a lidar ratio $R$ (also known as extinction-to-backscatter ratio) is required. This is defined as

$R=\frac{C_{\mathrm{ext}}}{C_{\mathrm{back}}}=\frac{k^{2} C_{\mathrm{ext}}}{S_{11}\left(180^{\circ}\right)}$,

where $C_{\text {back }}$ is the backscattering cross section - an indicator of the strength of backscattered $\left(\theta=180^{\circ}\right)$ intensity - and can be expressed as a function of $S_{11}\left(180^{\circ}\right)$. Linear depolarization ratio $\delta_{\mathrm{L}}$ (or LDR) describes the ability to depolarize backscattered light:

$\delta_{\mathrm{L}}=\frac{S_{11}\left(180^{\circ}\right)-S_{22}\left(180^{\circ}\right)}{S_{11}\left(180^{\circ}\right)+S_{22}\left(180^{\circ}\right)}$.

Lidar measurements of atmospheric depolarization are valuable in distinguishing traces of different particles because of the general sensitivity of the $S_{22}$ element to the particle shape; for isotropic, spherical particles, $S_{22}=S_{11}$ and $\delta_{\mathrm{L}}=0$.

\section{Mineral dust sample particles}

The Saharan mineral dust sample, from which the modelled particles were selected, was collected during the SAMUM campaign (Heintzenberg, 2009; Kandler et al., 2009) over Morocco $\left(30^{\circ} 17^{\prime} \mathrm{N}, 5^{\circ} 47^{\prime} \mathrm{W}, 3000 \mathrm{~m}\right.$ a.s.l.) on 6 June 2006 between 10:02 and 10:12 UTC (Universal time Coordinated) by an airborne cascade impactor particle collection system onto a carbon adhesive film (see Scheuvens et al., 2011 for details). For electron microscopy, the sample was sputter-coated with a thin gold layer. The single particles were imaged with an FEI environmental scanning electron microscope (ESEM) Quanta 200, equipped with a field emission gun, at different angles by tilting the specimen stage at a working distance of $10 \mathrm{~mm}$. Secondary and backscatter electron images were collected, and different combinations of acceleration voltages between $3 \mathrm{kV}$ and $30 \mathrm{kV}$ and "spot sizes" (beam currents) were tested. An acceleration voltage of $15 \mathrm{kV}$ with a "spot size 3" was found optimal for image quality, resulting in a nominal lateral resolution better than $3 \mathrm{~nm}$. In addition, characteristic X-ray fluorescence was measured with an energy-dispersive detector $(\mathrm{Si}(\mathrm{Li}))$. Elemental distribution within the particles was mapped with a resolution of 64 pixels $\times 50$ pixels (for Agg I, $128 \times 100$ ) per particle with a collection time of $0.2 \mathrm{~s}$ per pixel at an acceleration voltage of $25 \mathrm{kV}$ and "spot size 5". From the elemental distributions inside the particle and the morphological characteristics of the particle, a mineralogical composition was modelled by selecting minerals that best matched the measured elemental composition and morphology. For the single-scattering calculations, however, we had to select materials with known refractive indices from the literature and available databases, which matched most closely the mineral 
group and chemical composition. Birefringence of the minerals was omitted in this study because of the unknown optical axis directions and the limitations of the selected computational light-scattering method.

The particles chosen for this study are shown in Fig. 1 (left panels). They are named after their compositions which were determined from elemental maps obtained by energydispersive spectroscopy (example case shown in Fig. 2). Based on a mineralogical interpretation of the composition and characteristic shape features of each particle, we concluded the following.

- Cal I: a magnesium-bearing calcite particle with clay minerals at the edges. Based on Si and Al signals, these are addressed as a kaolinite group. In addition, two clear spots with high Fe content refer to iron oxides, and the $\mathrm{S}$ map shows some distinct locations with sulfate, which are taken as ammonium sulfate.

- Dol I: a dolomite particle with visible clay minerals. There is a larger attachment on the particle, which has a $\mathrm{Si}: \mathrm{Al}: \mathrm{K}(1: 0.8: 0.1$ atomic ratio) composition. This might be muscovite or illite. The morphology and $\mathrm{Al}: \mathrm{Si}$ atomic ratio of 0.5 of the smaller flakes refer to clay minerals, such as smectites (e.g. montmorillonite).

- Sil I: a magnesium-rich silicate particle; based on the crystal structure addressed as chrysotile. Three iron oxides adhering.

- Agg I: unlike the other particles, this does not have a main matrix with inclusions but is rather an agglomerate of several different species, most likely Nafeldspar, illite, quartz, and magnesium-rich clay. There are also small spots of iron oxides, two visible calcite grains, and an aluminum signal indicating possible smectites. See Fig. 2 for details.

Mineralogical information of the particles is gathered in Table 1 together with their estimated refractive indices at the wavelength $\lambda=550 \mathrm{~nm}$.

\section{Modelling approach}

The principal objective in this study was to derive the threedimensional particle shape and composition directly from measurements by combining stereogrammetry and mineralogical analysis of the particles, and compute the singlescattering properties for the retrieved dust particles. These phases are described in detail under the following subsections. For comparison, we also computed scattering by four corresponding simplified models, which are introduced here as well.

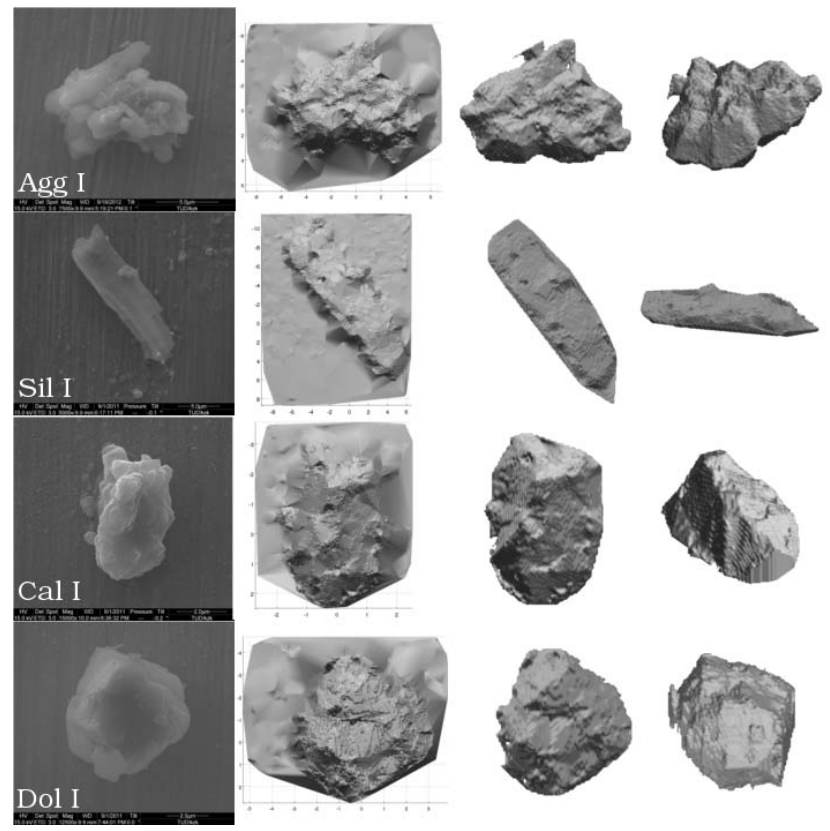

Fig. 1. Mineral dust particles used in this study (from left to right): scanning-electron microscope images, the retrieved TIN model of the surface, and the final volume representation from two different viewing angles.

\subsection{Stereogrammetric surface retrieval}

The surface topography of a dust particle was determined from a stereo pair of SEM images acquired by changing the tilt angle of the specimen stage between capturing the two images. The main task was to find automatically, for a set of points in the first image, the corresponding points in the second image. SIFT (scale invariant feature transform) keypoints (Lowe, 2004) were first extracted and matched to determine a sparse set of correspondences. In order to improve the SIFT matching, the SEM images were divided into subimages and the keypoint matching was performed within corresponding subimages. The subimages were 601 pixels squared in the first SEM image and somewhat larger in the second one. The positions of the corresponding subimages in the second image were also shifted according to rough horizontal and vertical disparities (single values for the whole image) visually estimated from the images. The small vertical disparities probably derived from marginal electron beam shifts due to the unavoidable charge build-up in the nonconductive particle. After SIFT matching, some manual editing was performed to remove correspondences which were clearly false matches. Horizontal and vertical disparities, i.e. differences between the image column coordinates and row coordinates of corresponding points were then computed and triangulated into sparse TIN (triangulated irregular network) models of disparities. 

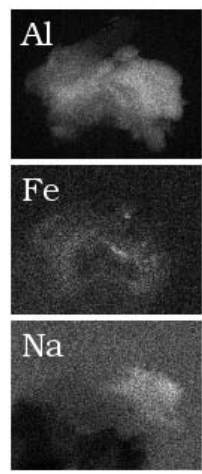
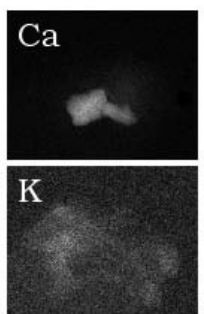

$\mathrm{O}$

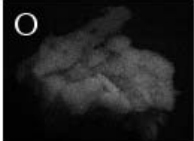

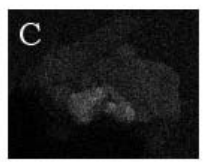

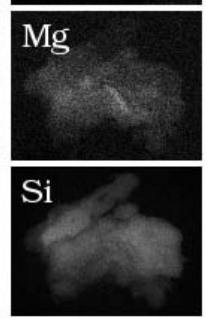

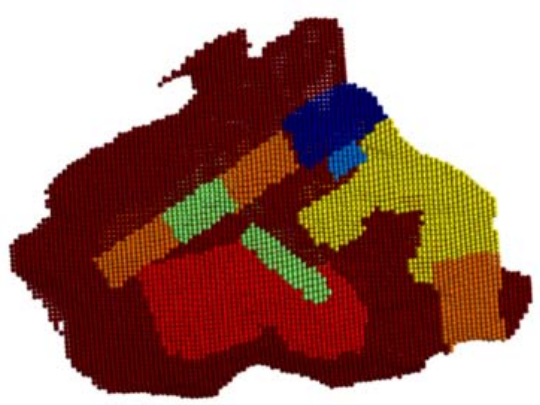

Fig. 2. Energy-dispersive spectroscopic images of the particle Agg I, based on which the elemental distribution and the composition of the particle were estimated (volume representation of the retrieved particle on the right). $\mathrm{O}$ and $\mathrm{Na}$ images show a pronounced shadowing effect towards the detector due to absorption of low-energy X-ray radiation inside the particle volume.

Table 1. Mineralogical composition of the selected dust particles, and respective refractive indices at $\lambda=550 \mathrm{~nm}$. MinDat stands for the web-based mineralogical database, http://www.mindat.org, where the information was retrieved in 2012.

\begin{tabular}{lrrl}
\hline Cal I & Volume fraction (\%) & Refractive index & Reference \\
\hline Calcite & 98 & 1.605 & Ghosh (1999) \\
Ammonium sulfate & 0.7 & 1.523 & Ivlev and Popova (1972) \\
Hematite & 0.7 & $3.102+i 0.0925$ & Longtin et al. (1988) \\
Kaolinite & 0.7 & 1.562 & MinDat \\
\hline Dol I & Volume fraction (\%) & Refractive index & Reference \\
\hline Dolomite & 97 & 1.622 & MinDat \\
Muscovite & 1.6 & 1.588 & MinDat \\
Montmorillonite & 1.3 & $1.523+i 3.82 \times 10^{-5}$ & Egan and Hilgeman (1979) \\
\hline Sil I & Volume fraction $(\%)$ & Refractive index & Reference \\
\hline Chrysotile & 98.0 & 1.57 & MinDat \\
Hematite & 2.0 & $3.102+i 0.0925$ & Longtin et al. (1988) \\
\hline Agg I & 66 & Refractive index & Reference \\
\hline Quartz & 11 & 1.546 & Longtin et al. (1988) \\
Calcite & 9.3 & 1.605 & Ghosh (1999) \\
Illite & 9.1 & 1.572 & MinDat \\
Albite & 2.0 & 1.535 & MinDat \\
Clinochlore & 1.8 & $1.523+i 3.82 \times 10^{-5}$ & Egan and Hilgeman (1979) \\
Montmorillonite & 1.5 & $3.102+i 0.0925$ & Longtin et al. (1988) \\
Hematite & & & \\
\hline & Volume fraction $(\%)$ &
\end{tabular}

The sparse set of correspondences was densified within the particle manually segmented from the first SEM image. The densification was based on affine least squares image matching carried out at interest points extracted from the first SEM image with the Canny edge detection algorithm (Canny, 1986). Around these edge points, there was sufficient variation in gray level for the image matching to perform appropriately. Let $\left(I_{1}, J_{1}\right)$ be an interest point in the first image, the approximate corresponding point of which is $\left(I_{2}^{0}, J_{2}^{0}\right)$ in the second image, obtained by linear interpolation from the sparse sets of disparities according to the disparity
TIN models. Let $g_{1}$ be a template image centred at $\left(I_{1}, J_{1}\right)$ and denote the pixels of the template by lower case letters $\left(i_{1}, j_{1}\right) \in \Omega$, where $\Omega$ is the domain of the template of size 61 pixels squared in the first image. The template image was matched against the second image $g_{2}$ within a search window centred at $\left(I_{2}^{0}, J_{2}^{0}\right)$. An affine transformation which maps the coordinates of the template image to the coordinates of the search image was defined as

$$
\begin{aligned}
\left(\begin{array}{c}
i_{12} \\
j_{12}
\end{array}\right) & =\left(\begin{array}{ll}
a_{1} & a_{2} \\
a_{3} & a_{4}
\end{array}\right)\left(\begin{array}{c}
i_{1}-I_{1}+I_{2}^{0} \\
j_{1}-J_{1}+J_{2}^{0}
\end{array}\right)+\left(\begin{array}{c}
a_{5} \\
a_{6}
\end{array}\right), \\
g_{12} & =g_{1}+a_{7},
\end{aligned}
$$


where $\mathbf{a}=\left[a_{1} \ldots a_{7}\right]^{T}$ were parameters to be estimated. The gray levels $g_{2}$ were bilinearly interpolated to the intermediate pixels $\left(i_{12}, j_{12}\right)$ of the transformed grid and denoted by $\tilde{g}_{2}$. The merit function to be minimized was given by

$$
\begin{aligned}
& f(\mathbf{a})=\sum_{\Omega}\left[w\left(i_{1}, j_{1}\right)\left(g_{12}\left(i_{1}, j_{1}\right)-\tilde{g}_{2}\left(i_{12}, j_{12}\right)\right)\right]^{2} / \\
& \sum_{\Omega}\left(w\left(i_{1}, j_{1}\right)\right)^{2}
\end{aligned}
$$

where $w$ are weights used to discard incompatible corresponding points, the gray levels of which differed more than an adaptive threshold, getting tighter as the iteration proceeded according to a scheme proposed by Zhang (1994). The nonlinear minimization problem was solved using the Levenberg-Marquardt algorithm. Once the iteration had converged, the refined corresponding point was obtained from

$$
\left(\begin{array}{l}
I_{2} \\
J_{2}
\end{array}\right)=\left(\begin{array}{ll}
a_{1} & a_{2} \\
a_{3} & a_{4}
\end{array}\right)\left(\begin{array}{c}
I_{2}^{0} \\
J_{2}^{0}
\end{array}\right)+\left(\begin{array}{l}
a_{5} \\
a_{6}
\end{array}\right) .
$$

The 3-D coordinates of the points on the surface of the particle were computed based on the established corresponding points between the SEM images. The pixel coordinates of the corresponding points were first transformed to metric units as $x_{1}=s\left(J_{1}-j_{0}\right), y_{1}=s\left(i_{0}-I_{1}\right)$ and $x_{2}=s\left(J_{2}-j_{0}\right), y_{2}=$ $s\left(i_{0}-I_{2}\right)$, where $s$ is a known scale and $\left(i_{0}, j_{0}\right)$ is the centre point of the SEM image. The 3-D coordinates were then given by (Themelis et al., 1990)

$$
\begin{aligned}
& X=\left(-x_{1} \sin \phi_{2}+x_{2} \sin \phi_{1}\right) / \sin \left(\phi_{1}-\phi_{2}\right) \\
& Y=\left(y_{1}+y_{2}\right) / 2 \\
& Z=\left(-x_{1} \cos \phi_{2}+x_{2} \cos \phi_{1}\right) / \sin \left(\phi_{1}-\phi_{2}\right),
\end{aligned}
$$

where $\phi_{1}$ and $\phi_{2}$ are the tilt angles around the $y$ axis of the specimen stage of the first and second SEM image, respectively. The 3-D points were finally triangulated into a TIN model.

Figure 3 gives a schematic presentation of the stereogrammetric method utilized in the shape retrieval of the dust particles. Figure 4 illustrates the SIFT keypoints and densified interest points on the first SEM image for which the corresponding points have been found from the second image. The horizontal disparities at each matched point are shown in Fig. 5. The geometry of the particle can be already perceived from this disparity image. The surface model of the particle is further shown in Fig. 1 (second column from the left). The SEM image has been rotated by 90 degrees in Figs. 4 and 5 to conform with the convention that the tilting occurs round the vertical $y$ axis.

\subsection{Three-dimensional structure and inhomogeneity}

The TIN model of the surface was easily transformed into volumetric 3-D form with interpolation. However, because the lower hemisphere of the particle was not imaged, some assumptions needed to be made of the shape and composition

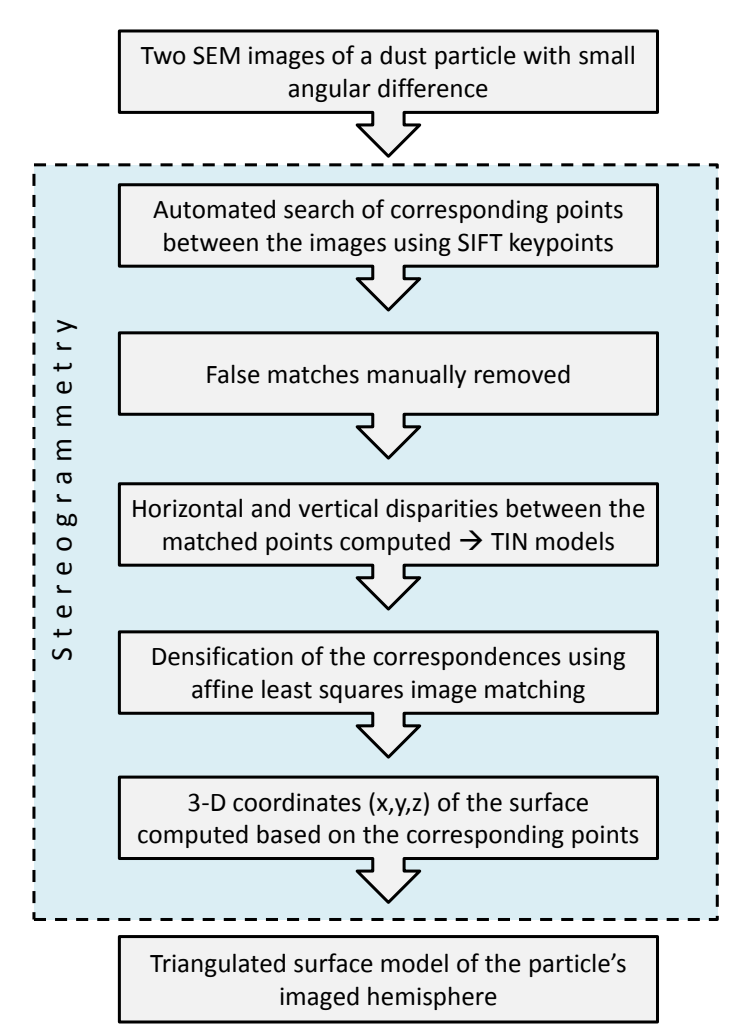

Fig. 3. A schematic presentation of the stereogrammetric shape retrieval method. A detailed description of each of the phases is given in Sect. 4.1.

at the backside of the particle. One approach was to form the other half by mirroring. For this, we needed to first establish which of the points were actually measured from the stage instead of the particle, setting the stage plane at $z=0$ and a mirroring plane at height $z=0.5 \cdot h$, where $h$ is the maximum height of the particle. We noted that mirroring worked well enough if less than $10 \%$ of the retrieved surface points were below the mirroring plane. If more than $10 \%$ of the surface points were found below $0.5 \cdot h$, too much of the surface information would have disappeared in mirroring and the known part of the shape altered excessively. In such cases, an alternative approach was used to find a lower mirroring plane located at the lowest $z$ value retrieved still above the stage plane. Then the mirrored points were scaled vertically to preserve the original aspect ratio of the particle. By using either of these two methods, mirroring or scaled mirroring, an entirely flat lower hemisphere could be avoided.

Details of the particle shape and inhomogeneity treatment are closely connected to, and partially dictated by, the lightscattering method chosen for the scattering computations. Of the publicly available methods, the discrete-dipole approximation was considered to be most suitable for the task. The computations were carried out using the parallelized version of the discrete-dipole approximation code ADDA 1.1 (Yurkin and Hoekstra, 2011). In the DDA, the particle is 


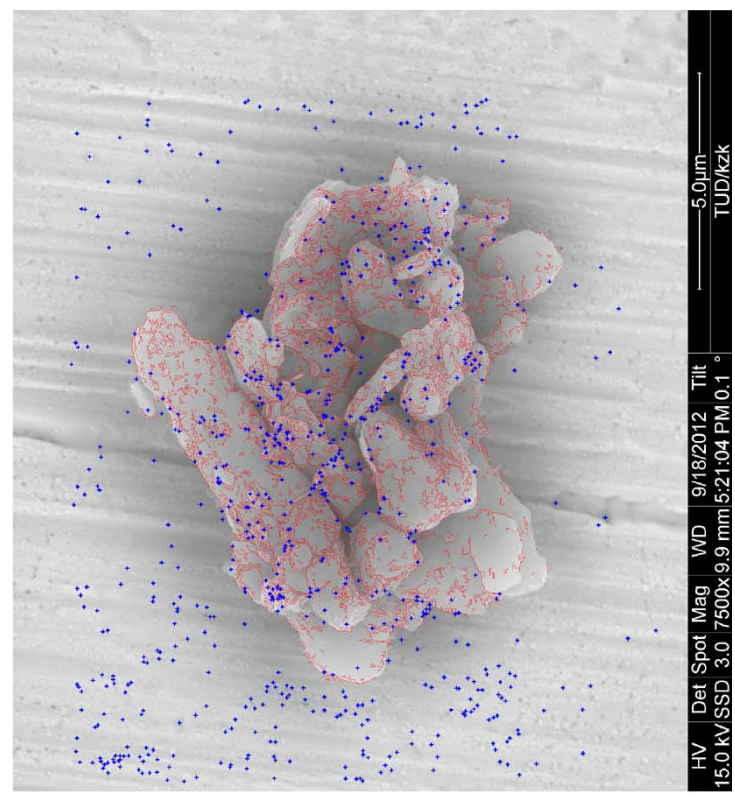

Fig. 4. Keypoints (blue crosses) and interest points (red dots) on the first SEM image of the Agg I particle.

presented with small, discrete volume elements which are treated as dipoles, and scattering is computed by integrating over the electric fields induced by these dipoles. Therefore, the resolution of the volume discretization, described with an interdipole separation $d$, dictates how small-scale morphological details can be considered. Also, the accuracy of the computed fields depends on $d$ : it should be small compared to the wavelength of radiation inside the particle. This criterion is often evaluated by the $|m| k d$ value but, according to Yurkin and Hoekstra (2011), no specific value that would guarantee a reasonable accuracy in all cases can be given; rather, they advice to first test the accuracy of the DDA in the desired situation to find the most suitable resolution. That is, as fine as needed for the accuracy, but no finer, to keep the computational demands feasible.

The volumes of the stereogrammetric shapes were discretized with approximately 100000 dipoles. This resolution was first used through size parameters $0.5-16$, resulting in $|m| k d \approx 0.90$ for the largest size parameter. Zubko et al. (2010) concluded for their irregular particles that $|m| k d \leq 1$ is an adequate accuracy criterion but, in our case, already $|m| k d \approx 0.90$ turned out to result in an insufficient accuracy with relative errors as high as $30 \%$ for $S_{44} / S_{11}$ at specific scattering angles. $S_{22} / S_{11}$ was also moderately sensitive. Consequently, the number of dipoles was increased for $x \geq 10$ by dividing each dipole into eight smaller dipoles, thus preserving the resolution of the morphological details through all size parameters. After this, the number of dipoles per wavelength was 22.7 and $|m| k d \approx 0.45$ for the largest size parameter $x=16$. With the chosen resolution, the running times of the parallelized computations varied from

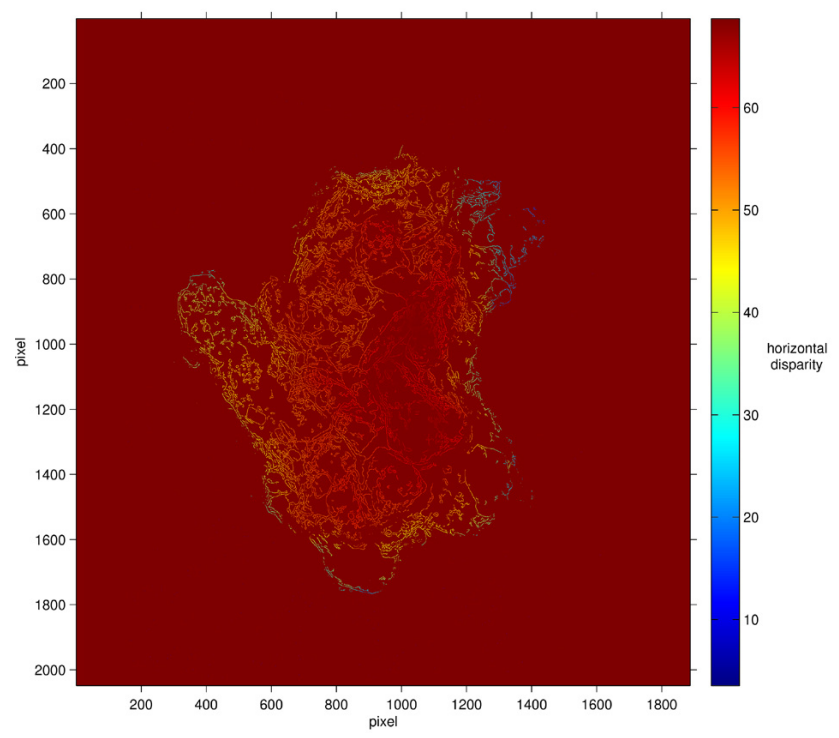

Fig. 5. Horizontal disparities reflecting the particle's feature elevation over the substrate plane at the matched points of the Agg I particle.

hours to a few days; the most demanding case was Sil I, which required $154 \mathrm{~h}$ on 46 processors, resulting in the total of $7084 \mathrm{~h}$ of CPU time.

The mineralogical interpretation of the elemental mappings revealed that all of the particles selected for this study were inhomogeneous. In ADDA, the polarizability of each dipole can be set separately, i.e. a complex refractive index value can be assigned to individual dipoles. Accordingly, for each particle, the spatial extents of different minerals were identified and refractive indices set. The depths of the inhomogeneities were estimated to equal the width, except in the case of clay-like minerals, which often present thin and flake-like shapes; for these, the depth was assumed to be only $20 \%$ of the maximum width. The connection between dipole polarizability and macroscopic refractive index was established in the computations using the lattice dispersion relation, which is the default setting in ADDA.

\subsection{Simplified models}

Because defining the composition of a dust particle in a predescribed way is not very efficient if the particle data sets are large, comparisons to scattering by homogeneous targets were also made to quantify the effect of inhomogeneity. An effective refractive index $m_{\text {eff }}$ was determined for each particle as a volume average of the permittivities of the component minerals (Chýlek et al., 2000).

In addition, the effects of shape on scattering were investigated using simplified shape models: a sphere, a spheroid, and a Gaussian random sphere. Volume equivalence was adopted to determine the corresponding sizes between the different shapes. The corresponding spheroidal model shape 


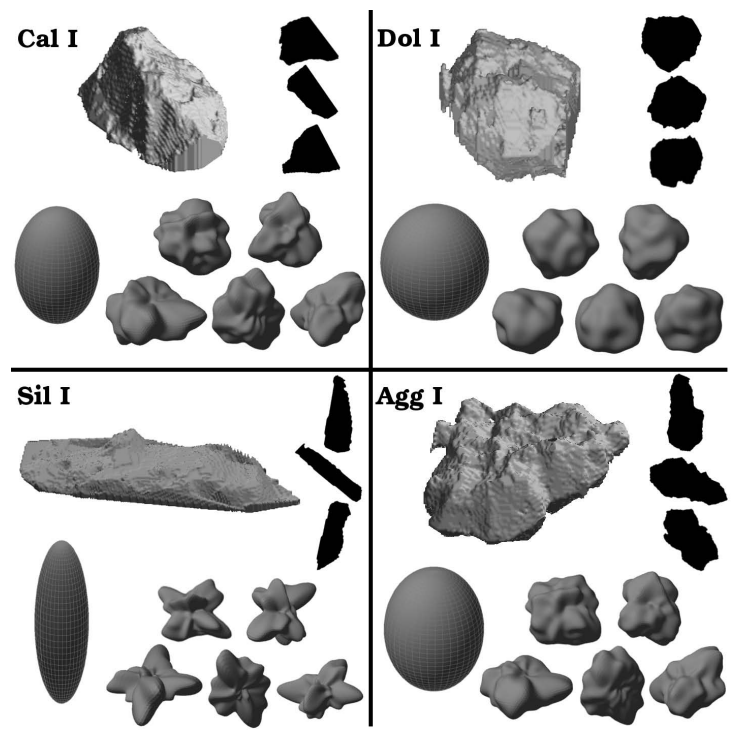

Fig. 6. Shapes of a prolate spheroid and five Gaussian random spheres derived as simplified models for each dust particle. The three black cross sections of each dust particle were used in deriving the corresponding Gaussian random sphere statistics.

Table 2. Effective refractive indices $m_{\text {eff }}$ and shape model parameters. The covariance functions for the Gaussian random sphere are not listed.

\begin{tabular}{lrrr}
\hline Particle & $m_{\text {eff }}$ & Aspect ratio & $\sigma$ \\
\hline Cal I & $1.619+i 0.00124$ & 0.6673 & 0.2802 \\
Dol I & $1.620+i 4.67 \times 10^{-7}$ & 0.9108 & 0.0835 \\
Sil I & $1.615+i 0.00355$ & 0.2918 & 0.5849 \\
Agg I & $1.589+i 0.00278$ & 0.7608 & 0.3377 \\
\hline
\end{tabular}

for each dust particle was derived by measuring the maximum diameter and the largest perpendicular width from the images directly from above. The ratio of the width to the diameter was used as the spheroidal aspect ratio, which are listed in Table 2. Because particle heights were closer to the width than to the maximum diameter, all model spheroids were chosen to be prolates, i.e. ellipses rotated about the maximum diameter. The model spheroids are illustrated with the respective dust particles in Fig. 6. Scattering by spheroids was computed with the T-matrix code by Mishchenko and Travis (1998).

The Gaussian random sphere is a statistical shape model for irregular particles introduced by Muinonen et al. (1996) and used in, e.g. scattering modelling of ice crystals (Sun et al., 2003). The model has proven to be extremely versatile considering that it is a function of two parameters only: the standard deviation of the radial distance $\sigma$ and the correlation function, which can further be combined as a covariance function. To apply the Gaussian random sphere geometry to our dust particles, their covariance functions were computed from three perpendicular, randomly chosen intersections for each particle (also illustrated in Fig. 6). The computational approach is explained in further detail by Nousiainen and McFarquhar (2004), who however used particle silhouettes rather than intersections. The derived $\sigma$ values are collected in Table 2. Because of the statistical nature of the Gaussian random sphere model, five (instead of one) sample shapes were generated for each dust particle, also shown in Fig. 6. The light-scattering properties were computed with ADDA and averaged over these five realizations.

Spherical equal-volume model particles were also considered, and their scattering was computed with the Mie code by Mishchenko et al. (2002).

\section{Results and discussion}

\subsection{Shape retrieval by stereogrammetry}

The performance of the stereogrammetric method was evaluated through visual inspection by comparing the retrieved shapes to anaglyph 3-D images of the same dust particles. Stereogrammetric shapes shown in Fig. 1 were generally quite well retrieved from the real dust particles. The method succeeded especially in reproducing particle aspect ratios and other large-scale features, e.g. surface height variation, but small-scale features could be identified to some extent only: for example, the flake near the lower-left corner in Dol I was only partially retrieved. In particular, the edges of the particles were challenging to distinguish from the background stage when the height separation was small, which was the case for Cal I and Sil I, both showing minor artifacts at the edges. Most importantly, it is inherently impossible to retrieve the internal structure of the particles by stereogrammetry. In our case, this deficiency is relevant particularly in the case of the agglomerate particle Agg I, which is supposedly not internally compact but is modelled as such through stereogrammetry.

\subsection{Monodisperse scattering}

The single-scattering simulations were carried out for four inhomogeneous stereogrammetric shapes and their homogeneous versions, as well as the corresponding simplified, homogeneous model particles of a sphere, a spheroid, and five Gaussian random spheres. All computations were performed for size parameters ranging from $x=0.5$ to $x=16$, with steps of 1 for $x<10$ and 2 otherwise. These $x$ correspond to equal-volume sphere radii $a_{\mathrm{eq}} \in[0.04,1.4] \mu \mathrm{m}$ at a wavelength $\lambda=550 \mathrm{~nm}$. Scattering results were averaged over 8704 particle orientations with the built-in orientation averaging scheme of ADDA, using the following number of different Euler angles: $\alpha=32, \beta=17$, and $\gamma=16$. The relative error of $C_{\text {ext }}$ resulting from orientation averaging was 
estimated to be below 0.015 for the highest size parameter considered.

From the orientation-averaged computations, we obtained the 10 scattering-matrix elements of Eq. (1). Of these, the dependence of $S_{11},-S_{12} / S_{11}, S_{22} / S_{11}, S_{33} / S_{11},-S_{34} / S_{11}$, and $S_{44} / S_{11}$ on particle size and scattering angle are presented in Fig. 7 for the four inhomogeneous stereogrammetric shapes. The elements $S_{13} / S_{11}, S_{14} / S_{11}, S_{23} / S_{11}$, and $S_{24} / S_{11}$ barely deviated from zero and, therefore, are not shown. These elements should, in fact, be exactly zero for mirror-symmetric particles. In our studies, Agg I is the only one that is truly mirror symmetric because also the inhomogeneities were mirrored in that case. Apparently, scaled mirroring and nonsymmetric surface inhomogeneities are not sufficient to cause any notable effects in the matrix elements for the other three shapes; however, the very minor deviations that were observed, were for particles with scaled mirroring (Cal I and Sil I).

As can be seen from Fig. 7, the six non-zero matrix elements for different particles show both similarities and differences. The $S_{11}$ element is strongly size-dependent and, except for Dol I, quite flat and featureless at side-scattering angles, peaking slightly towards backscattering. These are typical features of scattering by mineral dust. Dol I deviates from this at size parameters $x>4$ showing resonant structures visible as oscillations in the contour plots, and not just in $S_{11}$ but in all of the scattering-matrix elements. These resonances most probably originate from the equidimensionality of the particle: Dol I is closest to spherical while Sil I is the least spherical based on their shape parameters shown in Table 2 (sphericity quantified as aspect ratios close to unity and small $\sigma$ values). The resonances are the result of the complex interference of internal electromagnetic fields and have been discussed in more detail by Muinonen et al. (2011). The most elongated particle, Sil I, shows the most pronounced forward-scattering peak, with smaller relative contributions to side and backscattering.

For all of the particles, $-S_{12} / S_{11}$ is, at small $x$, very Rayleigh-like: symmetric and showing a positive maximum at $\theta=90^{\circ}$. As the particle diameter exceeds the wavelength, the phase differences between internal and external fields, as well as contributions from different parts of the particle to the internal field, become significant and redesign the scattering patterns, which now depend largely on the shape of the particle. Especially $-S_{12} / S_{11}$ and $S_{22} / S_{11}$ seem to be sensitive to particle shape for all $x>3$. The largest negative $-S_{12} / S_{11}$ is obtained for Dol I, again resembling scattering by spherical particles, while Sil I produces the largest positive values, and is also conserving the positive region at side scattering for large size parameters; whereas, for other particles, this gradually disappears below $x=6$. This could be an effect arising from the very elongated shape of Sil I: the physical dimensions of the particle remain small from most directions, possibly keeping the $-S_{12} / S_{11}$ positive. On the other hand, based on these results, also inhomogeneity and especially the absorbing constituents could promote positive $-S_{12} / S_{11}$, as the most positive values are seen for Agg I and Sil I, which contain $1.5-2 \%$ hematite.

The $S_{22} / S_{11}$ element deviates from unity already at very small size parameters $x<1$ in the case of the Sil I particle; whereas, for the same effect, four times that size is required in the case of Dol I. Cal I and Agg I show surprisingly similar size dependence of $S_{22} / S_{11}$ through all $x$, especially at side-scattering angles. $S_{22} / S_{11}$ is sometimes regarded as the indicative element of nonsphericity but it must be noted that the effect is not systematic: here, Sil I shows the strongest depolarization at small size parameters of all the particles, while for larger $x$ it becomes the weakest. Incidentally, Nousiainen et al. (2001) noted for microwave backscattering by small graupel particles that the largest-scale deviations from nonsphericity, related to elongation of the particle, were most efficient in depolarizing the backscattered radiation. Our findings here are consistent with this, also suggesting that when the size parameter increases, smaller-scale features in particles become relatively much more important for depolarization, which is quite reasonable. Interestingly, Dol I is the only shape showing a pronounced double minimum in $S_{22} / S_{11}$ at large scattering angles. This effect was called a double lobe by Lindqvist et al. (2009), who also reviewed some of the double lobes seen in other simulations and also measurements. Here, the phenomenon is not seen for Sil I, and remains quite weak for Cal I and Agg I, disappearing at large size parameters. Inhomogeneity and especially the presence of absorbing minerals might, at least partially, explain the disappearance of the double lobe: it was noted by Erkkilä (2008) that the lobe weakened systematically when the imaginary part of the refractive index was increased, although absorption was much higher in her case.

For $S_{33} / S_{11}$, Dol I shows behaviour that clearly differs from that for the other particle studies: the negative region is deep and becomes narrower as the size parameter increases. For the other particles, size dependence practically disappears at large $x$. Similarly, $-S_{34} / S_{11}$ is most different for Dol I. For the others, the differences are more subtle: for example, Sil I shows positive values at smaller scattering angles and lacks the positive region near backscattering at small sizes. Finally, $S_{44} / S_{11}$ is somewhat similar for Dol I, Cal I, and Agg I; the main differences being the resonances and the continuous negative region near backscattering present in the scattering by Dol I. It is also noteworthy that Sil I, the leastspherical particle, shows very similar size and angular dependence for both $S_{33} / S_{11}$ and $S_{44} / S_{11}$, the elements which are equal for spherical particles.

\subsection{Comparison to effective-medium particles}

The impact of particle inhomogeneity on scattering was studied by comparing the results for inhomogeneous particles to simulations where the mineralogical variation within one particle is described with an effective refractive index listed 

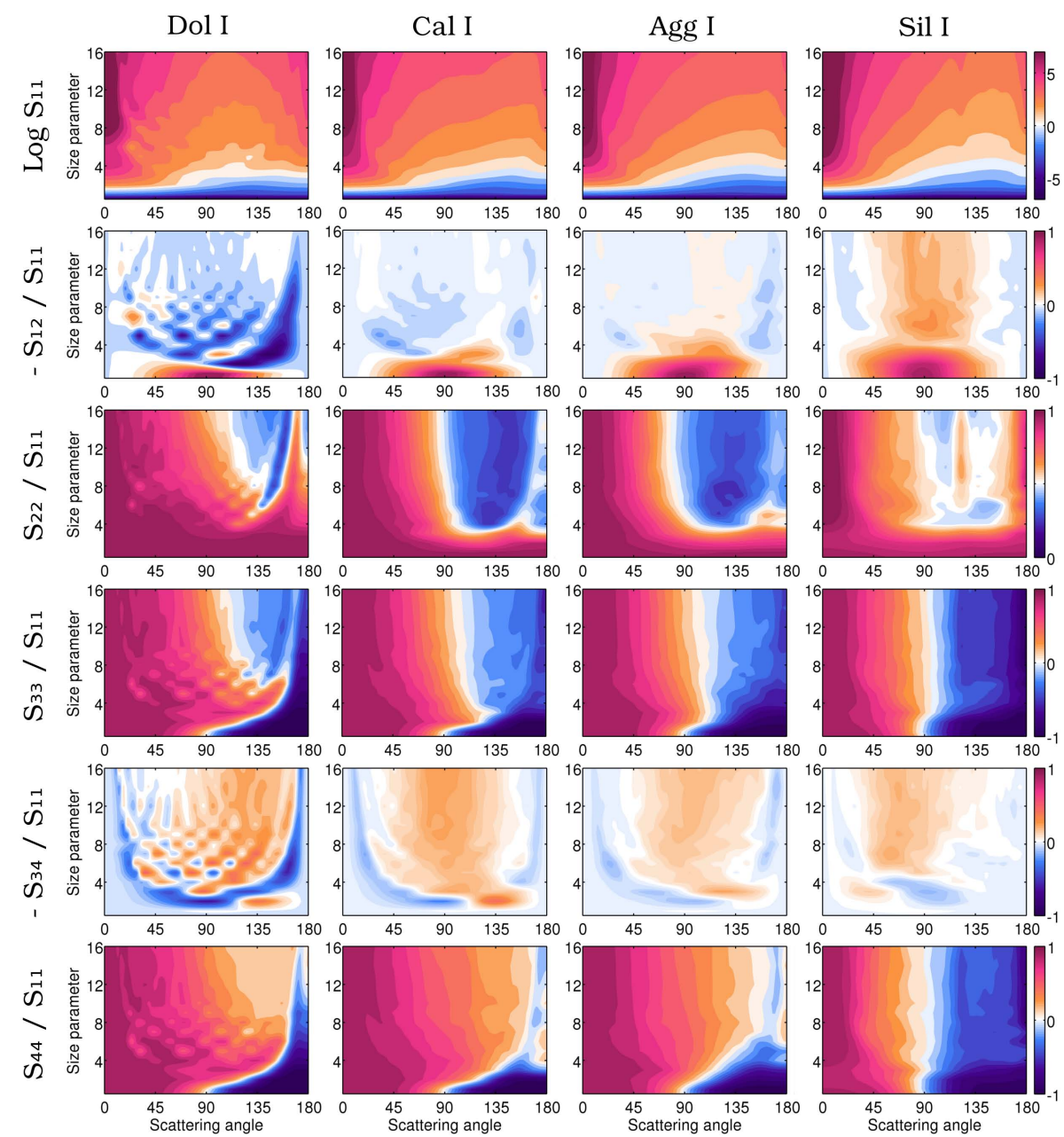

Fig. 7. Size and angular dependence of scattering by Dol I, Cal I, Agg I, and Sil I particles. The colour scales are presented on the right, separately for each matrix element.

in Table 2; these particles are, from here on, called homogeneous stereogrammetric shapes. The relative differences in the scattering-matrix elements between the inhomogeneous and homogeneous stereogrammetric shapes were quantified similarly to Nousiainen (2009), using slightly different definitions for the $S_{11}$ element and the element ratios:

$\Delta S_{11}=\frac{S_{11, \text { inhomog. }}-S_{11, \text { homog. }}}{S_{11, \text { inhomog. }}} \cdot 100$,

$\Delta S_{i j} / S_{11}=\left(\frac{S_{i j \text {,inhomog. }}}{S_{11 \text {,inhomog. }}}-\frac{S_{i j, \text { homog. }}}{S_{11, \text { homog. }}}\right) \cdot 100, \quad i j \neq 11$.

These are depicted as functions of the scattering angle and the size parameter for Agg I and Sil I in Figs. 8 and 9, respectively. For Cal I, the results appeared quite similar to Agg I but were more subtle and, for Dol I, hardly any differences could be observed. All this suggests that any differences seen may be mainly caused by the hematite content, due to its high real and imaginary parts of the refractive index. The most interesting observation in Figs. 8 and 9 is that there is quite a distinct size parameter region at $3<x<8$ where the effective-medium approximation most clearly over- or underestimates scattering. This is not even systematic for different particles: for example, $S_{33} / S_{11}$ and $S_{44} / S_{11}$ are being highly underestimated at large scattering angles roughly at $x=4$ for Sil I but overestimated for Agg I. Quite systematically, however, $S_{11}$ is overestimated near forward scattering at all size parameters and side scattering under- or overestimated, depending on the size parameter. By default, also, it seems that the effective-medium approximation overestimates $S_{22} / S_{11}$ at nearly all scattering angles when $x>3$.

\subsection{Size-integrated scattering}

Most practical considerations involve scatterers as a size distribution instead of monodisperse sizes. To consider such situations, we integrated scattering by each inhomogeneous 

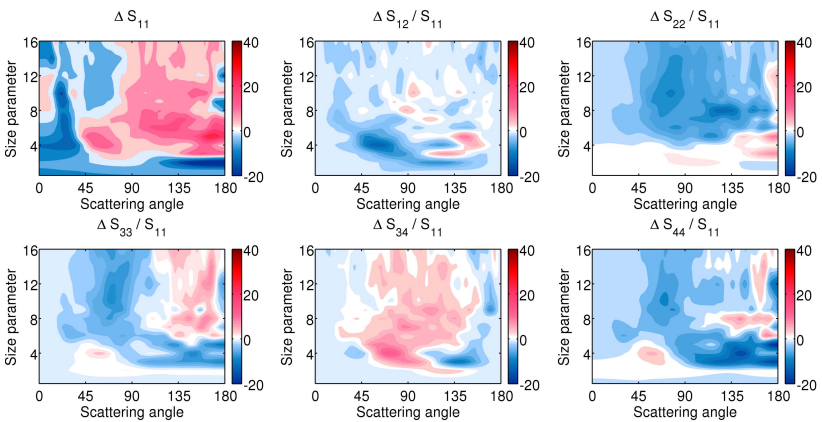

Fig. 8. Size dependence of the differences in the scattering-matrix elements between the inhomogeneous and homogeneous stereogrammetric shapes of Agg I.
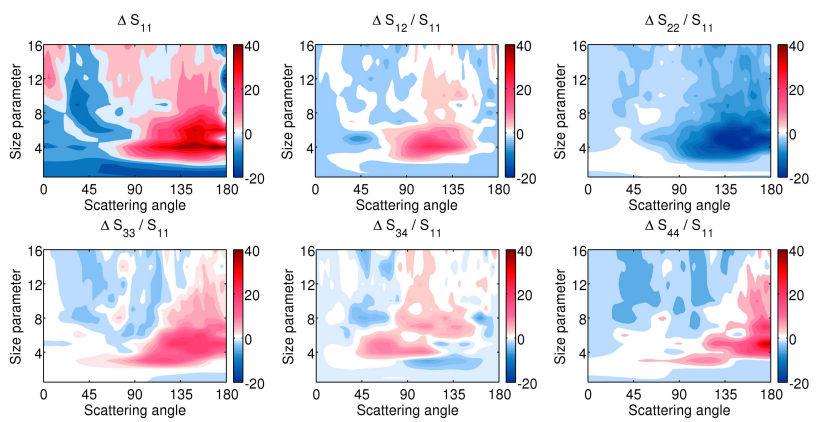

Fig. 9. Size dependence of the differences in the scattering-matrix elements between the inhomogeneous and homogeneous stereogrammetric shapes of Sil I.

stereogrammetric shape and the corresponding simplified model particles over a log-normal size distribution (Mishchenko and Travis, 1998):

$n(r)=\frac{1}{\sqrt{2 \pi} r \ln \sigma_{\mathrm{g}}} \exp \left(\frac{-\left(\ln r-\ln r_{\mathrm{g}}\right)^{2}}{2 \ln ^{2} \sigma_{\mathrm{g}}}\right)$,

where $r_{\mathrm{g}}=0.4 \mu \mathrm{m}$ and $\sigma_{\mathrm{g}}=2.0$. These parameters were chosen to have a fairly high $r_{\text {eff }}$, as representative of the atmospheric dust as possible with the size parameters considered, while keeping the form of the size distribution realistic, that is, the maximum concentration at small particle sizes. With these parameters, the cross-section-weighted average particle radius is $r_{\text {eff }}=0.82 \mu \mathrm{m}$. Our chosen size parameter range naturally covers only part of this example distribution.

The angular dependence of size-integrated scatteringmatrix elements for inhomogeneous and homogeneous stereogrammetric shapes are presented in Fig. 10, where also black lines are included to mark the scattering averaged over the four particles; an indication of what ensemble-averaged scattering of mineral dust could look like. This shows well that even though all the particles are mineral dust with fairly similar refractive indices, their single-scattering properties may vary to a notably large extent. Dust shape is crucial for scattering: for most elements, Sil I and Dol I show the largest differences and for them, differences in the physical dimensions of the particles are also the largest (Dol I being the most spherical and Sil I the least). However, depolarizationconnected $S_{22} / S_{11}$ is an interesting exception and shows that, even though this quantity is sensitive to particle shape, the shape dependence can be very complex.

\subsection{Comparison to simplified models}

The single-scattering properties of three simplified model shapes (sphere, spheroid, and Gaussian random sphere) were compared to the stereogrammetric shapes' results. Because of the large number of figures, or very busy ones, that would be needed to show their scattering matrices, we decided to instead focus on the scalar single-scattering properties, namely the asymmetry parameter $g$, single-scattering albedo $\varpi$, lidar ratio $R$, and linear depolarization ratio $\delta_{\mathrm{L}}$. These are shown in Fig. 11 for all of the particles considered. Overall, the parameters related to remote-sensing lidars $\left(R\right.$ and $\left.\delta_{\mathrm{L}}\right)$ are not well modelled here by any simplified model but, for the parameters significant in radiative flux estimations ( $g$ and $\varpi)$, better agreement is achieved in the case of all models other than spheres. All simplified models generally underestimated both $\varpi$ and $R$ of mineral dust; whereas $g$ and $\delta_{\mathrm{L}}$ were either overor underestimated, depending on the particle. The lidar ratio cannot be well modelled with spheres or spheroids because of their strong increase of intensity towards backscattering. Scattering by Dol I was seemingly easiest to fit using these three model geometries: all except $\delta_{\mathrm{L}}$ produced quite acceptable results. The scattering properties of the very thin and elongated Sil I particle, however, could not be well modelled by the simplified shape models considered. The differences between dust and homogeneous dust were in general small, mainly because the refractive indices of the different minerals had quite similar values, except for hematite. Estimated hematite content in all of the particles was small, not exceeding $2 \%$ of the volume. Nevertheless, when mixed with the other minerals for the effective refractive index, the locally absorbing inhomogeneities are spread throughout the particle, which easily causes biased absorption results, as in the case of Sil I single-scattering albedo in Fig. 11. Also, the linear depolarization ratio appears sensitive to absorption since, for the least-absorbing Dol I particle, $\delta_{\mathrm{L}}$ of the homogeneous dust agrees very well with that of the inhomogeneous dust but systematically larger discrepancies are noted for the particles containing small fractions of absorbing hematite. The size-integrated $S_{22} / S_{11}$ in Fig. 10, which is connected to $\delta_{\mathrm{L}}$, also supports this.

When the results are averaged over the four particles (solid lines in Fig. 11), the discrepancies between the inhomogeneous stereogrammetric shapes and the simplified models become generally smaller. Especially, nonsystematic differences tend to be balanced out and result in a better agreement, as is in the case of linear depolarization ratio for homogeneous stereogrammetric shapes or spheroids, and lidar ratio 

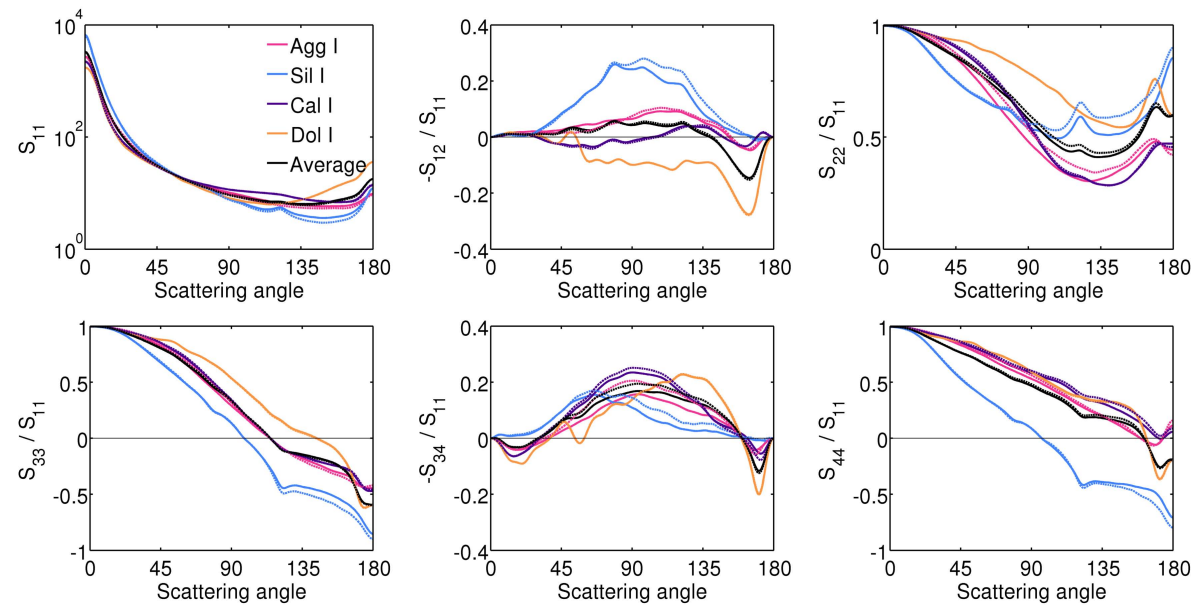

Fig. 10. Scattering results for a log-normal size distribution of inhomogeneous stereogrammetric shapes (solid lines). The effective-medium approximation results (homogeneous stereogrammetric shapes) are shown with dashed lines. Particle-averaged scattering properties are also presented.
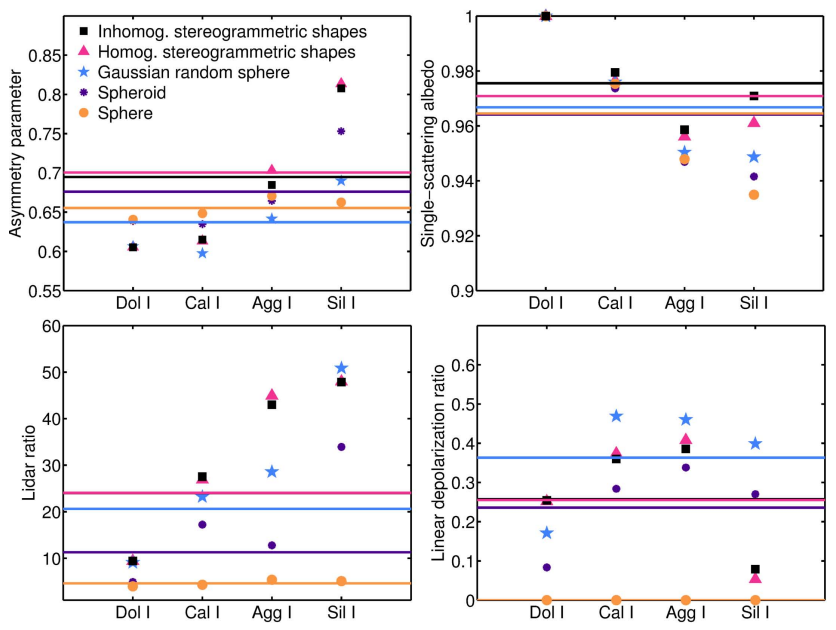

Fig. 11. Comparison of scattering properties of the inhomogeneous stereogrammetric shapes and simplified models: homogeneous stereogrammetric shapes, spheres, spheroids, and Gaussian random spheres. Scattering has been integrated over a log-normal size distribution with size parameters from $x=0.5$ to $x=16$. Solid lines correspond to the integrated results over the four particles. The top-panel parameters are important in radiation computations and climate considerations, and the bottom-panel parameters are needed in remote-sensing applications using a depolarization lidar.

for Gaussian random spheres. Through averaging, spheres even result here in a better agreement of the asymmetry parameter than the Gaussian random spheres. This is due to the systematic errors in $g$ by the Gaussian random spheres: in this case, the errors are preserved even after averaging. Same is true for $R$ and $\delta_{\mathrm{L}}$ of the average of spheres. In general, however, the results suggest that simplified models tend to work better when integrated over an ensemble - even as small as four particles - which justifies the use of simplified models in ensemble-averaged scattering but also reminds that the agreement is very dependent on the scattering by individual particles in the ensemble.

\section{Summary and conclusions}

The single-scattering properties of four Saharan mineral dust particles collected during the SAMUM campaign over Morocco in 2006 were computed, for the first time, using real shapes derived from the measured sample particles instead of assuming a mathematical, simplifying shape model. The three-dimensional shapes were retrieved from the scanningelectron microscope images using stereogrammetry. In addition, the spatial distribution of the mineralogical composition of the particles was derived by analysing the elemental mappings and the observable surface structures. One of the particles, Agg I, was an agglomerate of many minerals, and the other three were mostly composed of calcite (Cal I), dolomite (Dol I), or chrysotile (Sil I) but all included small amounts of other minerals. The shapes and the observed spatial distributions of the composition were then applied in singlescattering computations as is, using the well-established, semi-exact discrete-dipole approximation. Hence, scattering by these dust particles was computed as exactly as was possible. To investigate the size dependence of scattering, we considered size parameters $x=0.5-16$, which correspond to equal-volume sphere diameters between 0.08 and $2.8 \mu \mathrm{m}$ at a wavelength $\lambda=550 \mathrm{~nm}$.

The results showed great variation between the particles; from the size dependence of scattering and, especially, from scattering integrated over a log-normal size distribution, it was obvious that all of the dust types had very characteristic scattering properties and that these could not be accurately 
modelled by any single, fixed shape. The impact of particle inhomogeneity was studied by comparing the scattering properties of inhomogeneous stereogrammetric shapes with those of otherwise similar but homogeneous particles, for which an effective refractive index was determined by a mixing rule. Even though the resulting angular dependence of scattering was fairly similar, the single-scattering albedo decreased from 0.97 to 0.96 for the effective-medium Sil I particles. Absorption was largely due to hematite, although the total hematite content of the Sil I particle was as low as $2.0 \%$, which was nevertheless enough for causing a clear effect on the single-scattering albedo. Also, the linear depolarization ratio was nonsystematically affected when modelling hematite-containing dust with an effective refractive index. We can therefore conclude that effective-medium approximations should be used with extra care, also considering whether the change in the related parameter would be significant in the related application.

We also investigated how well scattering by the inhomogeneous stereogrammetric shapes considered could be reproduced using simplified model shapes of a sphere, a spheroid, and Gaussian random spheres. None of them generally succeeded in modelling light scattering by the inhomogeneous particles, even though the effective refractive index and shape parameters for other than spheres were derived for each particle individually. The lidar ratio and linear depolarization ratio, which are key parameters in remote-sensing applications using depolarization lidars, were found to be particularly challenging to model using these simplified shapes. Variation between different dust particles was considerable: the scattering properties of the very thin and flat Sil I particle proved to be challenging to model using any simplified shape but, then again, the properties of the more equidimensional Dol I could be considerably well reproduced by the Gaussian random spheres.

If the stereogrammetric shape-retrieval method could be improved to derive the complete three-dimensional particle shape by combining images taken from different sides of the particle, even the remaining assumptions about the geometry of the lower hemisphere would become unnecessary. Also, the method can distinguish and reproduce small-scale surface properties to some extent already but, since surface roughness is known to affect scattering quite notably, maybe this could even be improved. On the other hand, the discretedipole approximation used in light-scattering computations sets another limit for shape resolution, so the level of detail cannot be extensively increased without making the simulations unbearably heavy. To derive the internal structure of the dust particles, another potential morphological feature important for the single-scattering properties, it might be desirable to use the approach together with some method for investigating the particle interior, such as breaking up the particles after the stereogrammetric imaging.

The key results of this study were the accurate singlescattering properties for individual, inhomogeneous mineral dust samples and quantification of the particle-to-particle variability in scattering. The impact of inhomogeneity was also assessed based on observed spatial distribution of different component minerals. If applied to larger particle sets, the method presented here could be used to provide reference data for validating simpler shape models for mineral dust.

Acknowledgements. The authors wish to thank M. Yurkin and two anonymous referees for their constructive comments that helped to improve the manuscript. The authors acknowledge CSC - IT Center for Science Ltd. for providing the computational resources. M. Mishchenko and M. Yurkin are acknowledged for making their Mie, T-matrix, and ADDA codes publicly available. The work was partially funded by the Academy of Finland (contract 125180) and the Deutsche Forschungsgemeinschaft (DFG).

Edited by: Q. Fu

\section{References}

Berg, M. J. and Videen, G.: Digital holographic imaging of aerosol particles in flight, J. Quant. Spectrosc. Ra., 112, 1776-1783, 2011.

Bi, L., Yang, P., Kattawar, G., and Kahn, R.: Single-scattering properties of triaxial ellipsoidal particles for a size parameter range from the Rayleigh to geometric-optics regimes, Appl. Opt., 48, 114-126, 2009.

Bi, L., Yang, P., Kattawar, G., and Kahn, R.: Modeling optical properties of mineral aerosol particles by using nonsymmetric hexahedra, Appl. Opt., 49, 334-342, 2010.

Brock, R. S., Hu, X., Weidner, D. A., Mourant, J. R., and Lu, J. Q.: Effect of detailed cell structure on light scattering distribution: FDTD study of a B-cell with 3D structure constructed from confocal images, J. Quant. Spectrosc. Ra., 102, 25-36, 2006.

Canny, J.: A computational approach to edge detection, IEEE Trans. Pattern Anal. Machine Intell., 8, 679-698, 1986.

Chýlek, P., Videen, G., Geldart, D. J. W., Dobbie, J. S., and Tso, H. C. W.: Chapter 9: Effective medium approximations for heterogeneous particles, in: Light scattering by nonspherical particles, edited by: Mishchenko, M. I., Hovenier, J. W., and Travis, L. D., Academic Press, California, 2000.

Dubovik, O., Sinyak, A., Lapyonok, T., Holben, B. N., Mishchenko, M., Yang, P., Eck, T. F., Volten, H., Muñoz, O., Veihelmann, B., van der Zande, W. J., Leon, J.-F., Sorokin, M., and Slutsker, I.: Application of spheroid models to account for aerosol particle nonsphericity in remote sensing of desert dust, J. Geophys. Res., 111, D11208, doi:10.1029/2005JD006619, 2006.

Egan, W. G. and Hilgeman, T. W.: Optical properties of inhomogeneous materials, Academic, San Diego, California, 1979.

Erkkilä, H.: Light scattering by small inhomogeneous solar-system particles, Master's thesis, Observatory, University of Helsinki, 2008.

Formenti, P., Schütz, L., Balkanski, Y., Desboeufs, K., Ebert, M., Kandler, K., Petzold, A., Scheuvens, D., Weinbruch, S., and Zhang, D.: Recent progress in understanding physical and chemical properties of African and Asian mineral dust, Atmos. Chem. Phys., 11, 8231-8256, doi:10.5194/acp-11-8231-2011, 2011. 
Ghosh, G.: Dispersion-equation coefficients for the refractive index and birefringence of calcite and quartz crystals, Opt. Commun., 163, 95-102, 1999.

Goudie, A. S. and Middleton, N.: Desert dust in the global system, Springer, 2006.

Heintzenberg, J.: The SAMUM-1 experiment over Southern Morocco: overview and introduction, Tellus B, 61, 2-11, 2009.

$\mathrm{Hu}, \mathrm{R}$. M. and Sokhi, R. S.: Light scattering and absorption properties of dust particles retrieved from satellite measurements, J. Quant. Spectrosc. Ra., 110, 1698-1705, 2009.

Ishimoto, H., Zaizen, Y., Uchiyama, A., Masuda, K., and Mano, Y.: Shape modeling of mineral dust particles for light-scattering calculations using the spatial Poisson Voronoi tessellation, J. Quant. Spectrosc. Ra., 111, 2434-2443, 2010.

Ivlev, L. S. and Popova, S. I.: Optical constants of substances of atmospheric aerosol, Izv. Vuz. Fiz., 5, 91-97, 1972.

Jacobs, K. M., Lu, J. Q., and Hu, X.-H.: Development of a diffraction imaging flow cytometer, Opt. Lett., 34, 2985-2987, 2009.

Kandler, K., Schütz, L., Deutscher, C., Hofmann, H., Jäckel, S., Knippertz, P., Lieke, K., Massling, A., Schladitz, A., Weinzierl, B., Zorn, S., Ebert, M., Jaenicke, R., Petzold, A., and Weinbruch, S.: Size distribution, mass concentration, chemical and mineralogical composition, and derived optical parameters of the boundary layer aerosol at Tinfou, Morocco, during SAMUM 2006, Tellus, 61, 32-50, 2009.

Kaye, P. H., Aptowicz, K., Chang, R. K., Foot, V., and Videen, G.: Angularly resolved elastic scattering from airborne particles, in: Optics of Biological Particles, edited by: Hoekstra, A. G., Maltsev, V. P., and Videen, G., Springer, Dordrecht, 31-61, 2007.

Lindqvist, H., Muinonen, K., and Nousiainen, T.: Light scattering by coated Gaussian and aggregate particles, J. Quant. Spectrosc. Ra., 110, 1398-1410, 2009.

Longtin, D. R., Shettle, E. P., Hummel, J. R., and Pryce, J. D.: A wind dependent desert aerosol model: Radiative properties, AFGL-TR-88-0112, Air Force Geophysics Laboratory, Hanscom AFB, MA, 1988.

Lowe, D.: Distinctive image features from scale-invariant keypoints, Int. J. Comput. Vision, 60, 91-110, 2004.

Macke, A., Mueller, J., and Raschke, E.: Single scattering properties of atmospheric ice crystals, J. Atmos. Sci., 53, 2813-2825, 1996.

Merikallio, S., Lindqvist, H., Nousiainen, T., and Kahnert, M.: Modelling light scattering by mineral dust using spheroids: assessment of applicability, Atmos. Chem. Phys., 11, 5347-5363, doi:10.5194/acp-11-5347-2011, 2011.

Mishchenko, M. I. and Travis, L. D.: Capabilities and limitations of a current FORTRAN implementation of the T-matrix method for randomly oriented, rotationally symmetric scatterers, J. Quant. Spectrosc. Ra., 60, 3, 309-324, 1998.

Mishchenko, M. I., Travis, L. D., and Lacis, A. A.: Scattering, absorption, and emission of light by small particles, Cambridge University Press, Cambridge, 2002.

Mishra, S. K., Tripathi, S. N., Aggarwal, S. G., and Arola, A.: Optical properties of accumulation mode, polluted mineral dust: effects of particle shape, hematite content and semiexternal mixing with carbonaceous species, Tellus B, 64, 18536, doi:10.3402/tellusb.v64i0.18536, 2012.

Muinonen, K., Nousiainen, T., Fast, P., Lumme, K., and Peltoniemi, J. I.: Light scattering by Gaussian random particles: Ray optics approximation, J. Quant. Spectrosc. Ra., 55, 577-601, 1996.
Muinonen, K., Tyynelä, J., Zubko, E., Lindqvist, H., Penttilä, A., and Videen, G.: Polarization of light backscattered by small particles, J. Quant. Spectrosc. Ra., 112, 2193-2212, 2011.

Muñoz, O., Moreno, F., Guirado, D., Dabrowska, D. D., Volten, H., and Hovenier, J. W.: The Amsterdam-Granada light scattering database, J. Quant. Spectrosc. Ra., 113, 565-574, 2012.

Nousiainen, T.: Optical modeling of mineral dust particles: a review, J. Quant. Spectrosc. Ra., 110, 1261-1279, 2009.

Nousiainen, T., Muinonen, K., Avelin, J., and Sihvola, A.: Microwave backscattering by nonspherical ice particles using second-order perturbation series, J. Quant. Spectrosc. Ra., 70, 639-661, 2001.

Nousiainen, T., Muinonen, K., and Räisänen, P.: Scattering of light by large Saharan dust particles in a modified ray optics approximation, J. Geophys. Res., 108, 4025, doi:10.1029/2001JD001277, 2003.

Nousiainen, T. and McFarquhar, G. M.: Light scattering by quasispherical ice crystals, J. Atmos. Sci., 61, 2229-2248, 2004.

Nousiainen, T., Kahnert, M., and Lindqvist, H.: Can particle shape information be retrieved from light-scattering observations using spheroidal model particles?, J. Quant. Spectrosc. Ra., 112, 22132225, 2011a.

Nousiainen, T., Muñoz, O., Lindqvist, H., Mauno, P., and Videen, G.: Light scattering by large Saharan dust particles: comparison of modeling and experimental data for two samples, J. Quant. Spectrosc. Ra., 112, 420-433, 2011 b.

Nousiainen, T., Zubko, E., Lindqvist, H., Kahnert, M., and Tyynelä, J.: Comparison of scattering by different nonspherical, wavelength-scale particles, J. Quant. Spectrosc. Ra., 113, 23912405, 2012.

Orlova, D. Y., Yurkin, M. A., Hoekstra, A. G., and Maltsev, V. P.: Light scattering by neutrophils: model, simulation, and experiment, J. Biomed. Opt., 13(5), 054057, doi:10.1117/1.2992140, 2008.

Reid, J. S., Reid, E. A., Walker, A., Piketh, S., Cliff, S., Al Mandoos, A., Tsay, S.-C., and Eck, T. F.: Dynamics of southwest Asian dust particle size characteristics with implications for global dust research, J. Geophys. Res., 113, D14212, doi:10.1029/2007JD009752, 2008.

Scheuvens, D., Kandler, K., Küpper, M., Lieke, K., Zorn, S. R., Ebert, M., Schütz, L., and Weinbruch, S.: Individual-particle analysis of airborne dust samples collected over Morocco in 2006 during SAMUM 1, Tellus B, 63, 512-530, 2011.

Strokotov, D. I., Moskalensky, A. E., Nekrasov, V. M., and Maltsev, V. P.: Polarized light-scattering profile - advanced characterization of nonspherical particles with scanning flow cytometry, Cytometry, 79, 570-579, 2011.

Sun, W., Loeb, N. G., Videen, G., and Fu, Q.: Examination of surface roughness on light scattering by long ice columns by use of a two-dimensional finite-difference time domain algorithm, Appl. Opt., 43, 1957-1964, 2004.

Sun, W., Nousiainen, T., Muinonen, K., Fu, Q., Loeb, N. G., and Videen, G.: Light scattering by Gaussian particles: A solution with finite-difference time domain technique, J. Quant. Spectrosc. Ra., 79-80, 1083-1090, 2003.

Themelis, G., Chikwembani, S., and Weertman, J.: Determination of the orientation of $\mathrm{Cu}-\mathrm{Bi}$ grain boundary facets using a photogrammetric technique, Mater. Charact., 24, 27-40, 1990. 
van de Hulst, H. C.: Light scattering by small particles, 2nd ed., Dover, 1981.

Yurkin, M. A. and Hoekstra, A. G.: The discrete-dipoleapproximation code ADDA: capabilities and known limitations, J. Quant. Spectrosc. Ra., 112, 2234-2247, 2011.
Zhang, Z.: Iterative point matching for registration of free-form curves and surfaces, Int. J. Comput. Vision, 13, 119-152, 1994.

Zubko, E., Petrov, D., Grynko, Y., Shkuratov, Y., Okamoto, H., Muinonen, K., Nousiainen, T., Kimura, H., Yamamoto, T., and Videen, G.: Validity criteria of the discrete dipole approximation, Appl. Opt., 49, 1267-1279, 2010. 